\title{
O USO DE GEOTECNOLOGIAS NO PLANEJAMENTO AMBIENTAL DE UNIDADES DE CONSERVAÇÃO: ESTUDO DE CASO RESERVA BIOLÓGICA DAS ARAUCÁRIAS
}

\author{
Ronaldo Ferreira Maganhotto $^{(a)}$, Luiz Claudio de Paula Souza ${ }^{(b)}$, Jairo Calderari Oliveira \\ Junior $^{(\mathrm{c})}$, MarcielLohmann ${ }^{(\mathrm{d})}$
}

\author{
(a) Turismo/UNICENTRO, ronaldomaganhotto@gmail.com \\ (b) Agronomia/UFPR, lcsouza@ufpr.br \\ (c) Agronomia/UTFPR, jairo@agronomo.eng.br \\ (d) Geografia/UEL, marciel_lohmann@hotmail.com
}

\section{Eixo: GEOTECNOLOGIAS E MODELAGEM ESPACIAL EM GEOGRAFIA FÍSICA}

\section{Resumo}

\begin{abstract}
Frente a necessidade de dados quantitativos fundamentando uma avaliação ambiental sistêmicapretende-se demonstrar a utilização dos Índices de Representação do Relevo e aplicação da Lógica Fuzzy no planejamento ambiental da REBIO das Araucárias. Localizada na Região Centro Sul do Paraná, a unidade não dispõe de Plano de Manejo, nem de Zoneamento Ambiental. O processamento de atributos topográficos como: Altitude AboveChannel Network, Wetness Index, Multiresolution Index of Valley BottomFlatness, Slope e Ls Factor, resultou na seguimentação da unidade em Classes Limitantes. A maior parte da REBIO encontra-se disposta nas Classes de Limitação Muito Baixa e Baixa, abrangendo, respectivamente, cerca de 49 e $21,07 \%$ da unidade. Desta forma, afirma-se que cerca de $70 \%$ da unidade correspondem a porções territoriais passíveis de um uso menos limitante, uma vez que, estas áreas não estão associadas a intensos processos erosivos, sedimentares e de inundação.
\end{abstract}

Palavras chave: Geotecnologias, Atibutos Topográficos, Fuzzy, REBIO.

\section{Geotecnologias e Sua Aplicação no Planejamento Ambiental}

O planejamento ambiental, fundamentado em uma visão sistêmica, deve vir a orientar a ação antrópica sob o meio. Para Ross (2006, p. 59), a correlação das informações resulta na identificação de espaços territoriais a que se pode denominar de unidades de paisagens, unidades ambientais ou ainda sistemas ambientais, com base nos quais se formula o entendimento das fragilidades potenciais e emergentes, bem como as potencialidades dos recursos naturais e as potencialidades humanas dos grupos sociais que habitam esses espaços, ou esses lugares.

Neste contexto, o presente trabalho apresenta o processamento dos Índices de Representação do Relevo (IRR), e a Aplicação da Lógica Fuzzy, para fins de planejamento da Reserva Biológica das Araucárias, unidade de conservação, localizada nos municípios de Imbituva, Fernandes Pinheiro e Teixeira Soares.

A unidade de 14.980 ha, criada em 23 de Março de 2006, apresenta uma série de dificuldades como: pendências na regulamentação fundiária, recursos financeiros e a inexistência de plano de manejo. 


\section{OS DESAFIOS DA GEOGRAFIA FÍSICA NA FRONTEIRA DO CONHECIMENTO \\ Instituto de Geociências - Unicamp \\ Campinas - SP \\ 28 de Junho à 02 de Julho de 2017}

Assim o processamento destas informações devem vir contribuir para o planejamento e manejo da área.

Para Maganhotto et al. (2016) os índices Altitude AboveChannel Network (AACN), Wetness Index (WI), Multiresolution Index of Valley BottomFlatness (MRVBF), Slope e Ls Factor, podem ser associados as características ambientais físicas de determinada área.

De posse destas informações, verificou-se que os valores destes atributos apresentam-se em diferentes escalas, dificultando o cruzamento destas informações. Entretanto, este obstáculo pode ser contornado com a utilização da lógica Fuzzy, ferramenta que possibilita a padronização das informações.

A utilização do Geoprocessamento em análises ambientais, espacializa e quantifica as variáveis físicas, o processamento dos dados neste ambiente possibilita a sobreposição e cruzamento das informações favorecendo a obtenção de mapas sintéticos e nas tomadas de decisão para a resolução ou minimização de problemas ambientais (CARVALHO FILHO e XAVIER DA SILVA,1995).

\section{O processamento dos Índices de Representação do Relevo na REBIO das Araucárias.}

A utilização dos Índices de Representação do Relevo, IRR, como instrumento de análise ambiental, ganhou destaque na década de 90. A possibilidade de uma avaliação em ambiente computacional, a partir do Modelo Numérico do Terreno, MNT, e correlação dos mesmos com a geomorfologia e seus processos, impulsionaram sua aplicação às pesquisas ambientais (MOORE et al., 1993; IPPOLITI, 2005).

Segundo Maganhoto et al. (2013), os IRR que melhor representam a fragilidade ambiental são: Altitude em Relação ao Canal de Drenagem (Altitude AboveChannel Network - AACN), Índice de Umidade (TopographicWetness Index - TWI), Declividade (Slope), Potencial de Erosão e Sedimentação (Multiresolution Index of Valley BottomFlatness - MRVBF) e Relação do Comprimento de Rampa e Declividade (LS).

O AACN representa a distância vertical da célula em relação à célula mais próxima. Valores pequenos de AACN indicam locais em que o lençol freático pode estar mais próximo da superfície do solo, caracterizando zonas de acumulação (BÖHNER et al., 2002). Os valores intermediários indicam zonas de transferência de material (encostas), enquanto valores maiores indicam condições mais elevadas da superfície geomórfica (possíveis zonas de perda de material) (BÖHNER et al., 2002). A Figura 1 apresenta a espacialização e a distribuição das classes deste atributo na REBIO. 


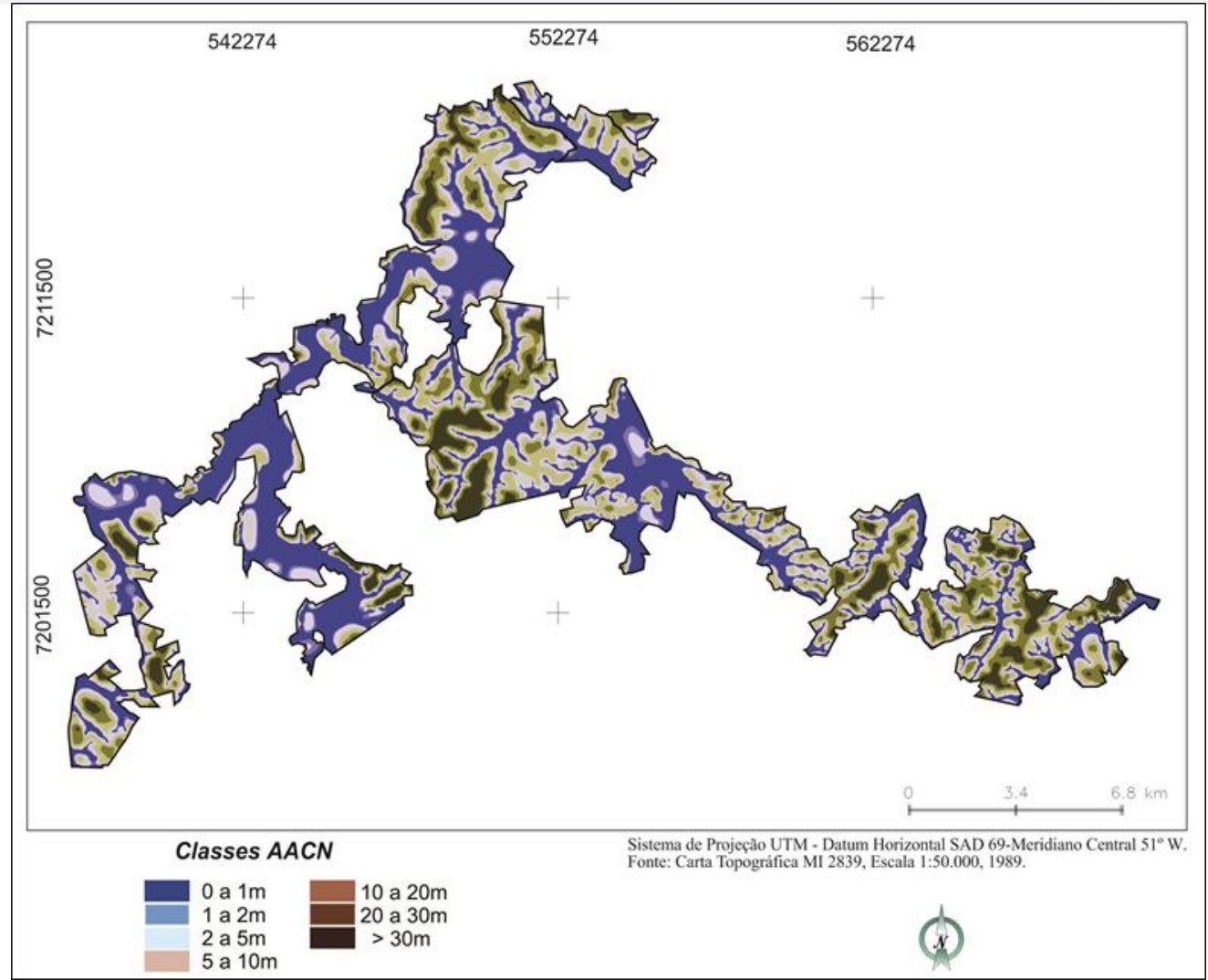

Figura 1: AACN REBIO

Organizado: Maganhotto, R.F.

Pode-se afirmar que 7.484 ha, área correspondente a 50\% da unidade, encontram-se inseridos no intervalo de 0 a 5 metros de distância da drenagem sob sua influência direta. Os 50\% restantes situamse a uma distância mínima de 5 metros da drenagem, configurando-se como porções territoriais menos suscetíveis a inundações.

O TWI, descreve a tendência de uma célula acumular água. Assim, os maiores valores de TWI indicam maior tendência de acumular água e, portanto, maior conteúdo de água no solo. (GRUBER; PECKHAM, 2009). A Figura 2 apresenta a espacialização e a distribuição das classes deste atributo na REBIO.

Baseando-se nos parâmetros determinados por Prates (2010) e Lin et al. (2006), pode-se afirmar que cerca de1850 ha, 55\% da unidade, correspondem a superfícies mal drenadas associadas a valores de TWI maiores que 8. As demais áreas situam-se em condições de boa drenagem, registrando valores abaixo de 8 . 


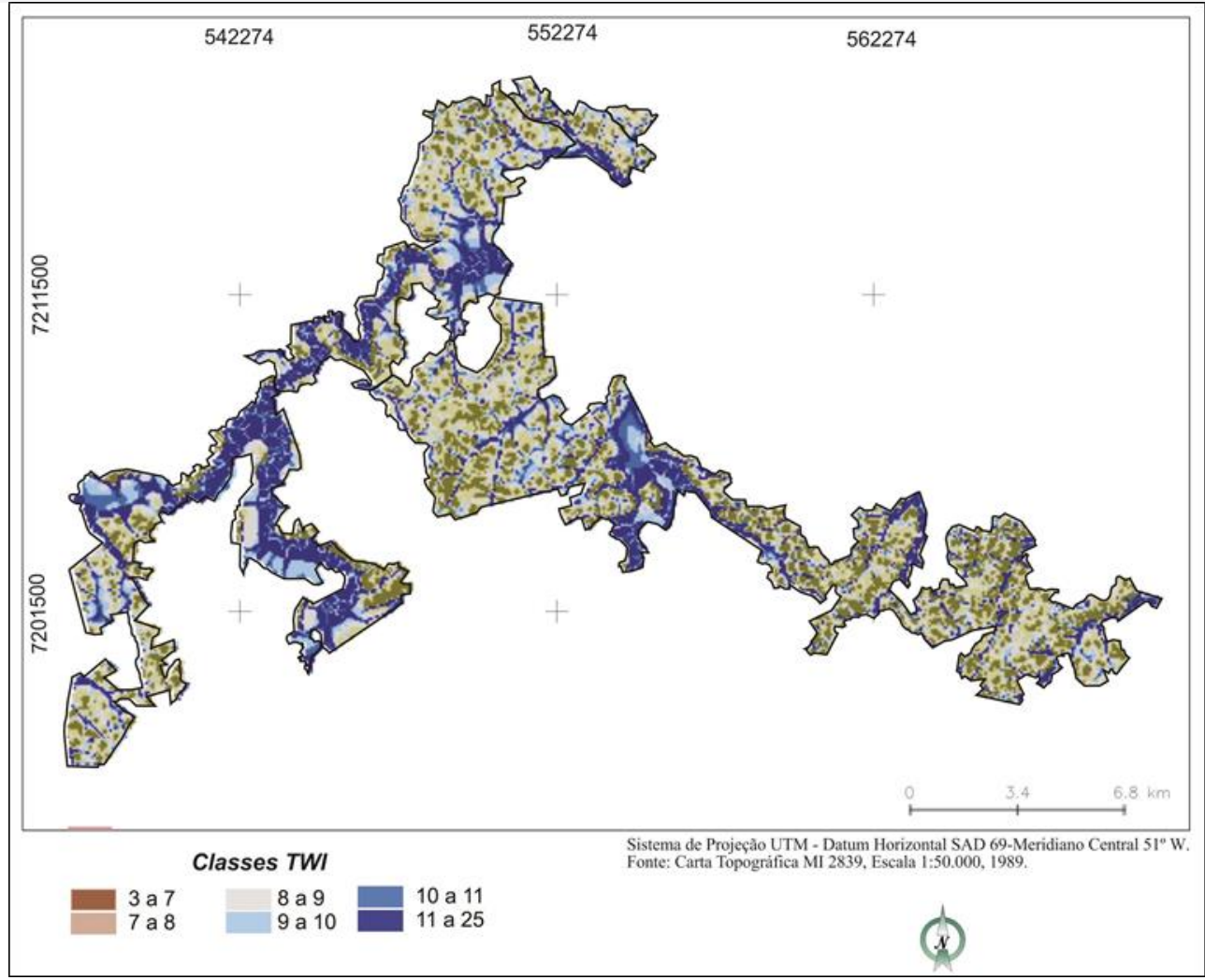

Figura 2: TWI REBIO

Organizado: Maganhotto, R.F.

O MRVBF, foi projetado para mapear áreas de sedimentação e deposição na paisagem. Para Mckergow et al. (2007) locais com MRVBF menor que 0,5 reportam-se às encostas; valores maiores que 0,5 e menores que 1 correspondem a pequenos fundos de vale; e maiores que 1 a fundos de vale maiores. A Figura 3 apresenta a espacialização e a distribuição das classes deste atributo na REBIO.

Correlacionando as informações tabuladas com os parâmetros definidos por Mckenzie e Gallant (2007) para o referido atributo, pode-se afirmar a ocorrência de erosão em, aproximadamente, 5580 ha, 37\% da FLONA inseridos no intervalo de 0 a 0,5. Registrou-se, também, sedimentação para as áreas caracterizadas por valores acima de 2,5, presentes em, 4525 ha, equivalentes a $30 \%$ da unidade. Os 33\% restantes distribuem-se em áreas livres de processos erosivos e de sedimentação.

O Slope é definida por Burrough (1986) como sendo um plano tangente à superfície, expresso como a mudança de elevação sobre certa distância, normalmente calculada em graus ou em porcentagem. A Figura 4 apresenta a espacialização e a distribuição das classes deste atributo na REBIO. 


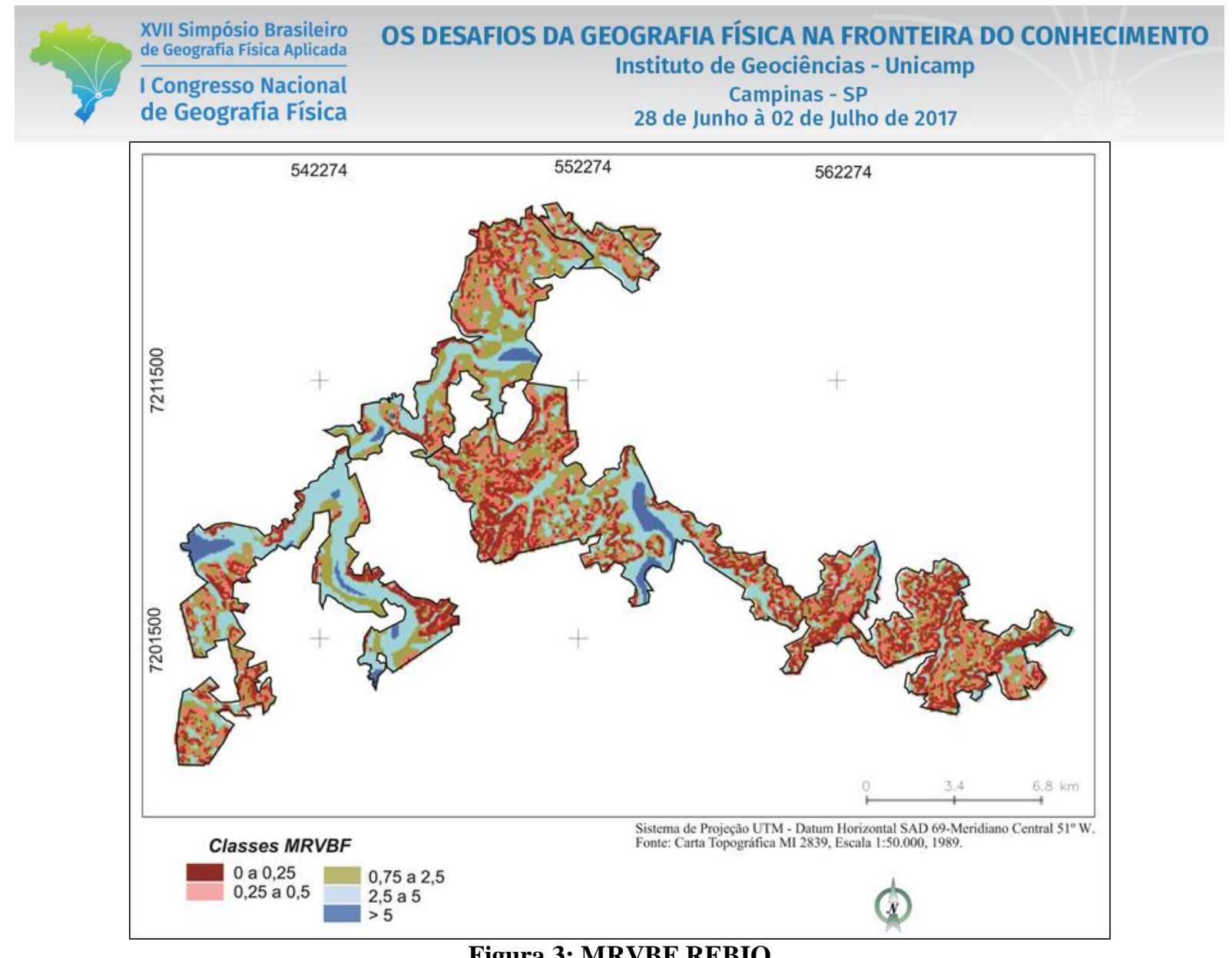

Figura 3: MRVBF REBIO

Verificou-se com a caracterização deste atributo que $13 \%$ da unidade, encontram-se em declividades maiores que $15 \%$, necessitando de maiores cuidados devido sua propensão aos processos erosivos.

O LS Factor representa o efeito da topografia sobre a erosão (quanto maior o LS, maior o potencial erosivo), e reproduz o efeito combinado do comprimento e grau de declive da encosta (BERTONI e LOMBARDI-NETO, 1990). A Figura 5 apresenta a espacialização e a distribuição das classes deste atributo na REBIO.

A quantificação deste atributo possibilitou a identificação de áreas suscetíveis aos processos erosivos. Cerca de, 4033 ha da unidade, ou seja, 27\% registraram Ls Factor, acima de 4, valor definido por Mansor et al. (2002) como indicativo de áreas potencialmente erosivas.

Com base nestes dados, na fundamentação teórica e no trabalho de campo, evidencia-se a possibilidade de associar estas informações a condição geomorfológica, pedológica e de fragilidade/susceptibilidade ambiental da REBIO das Araucárias. 


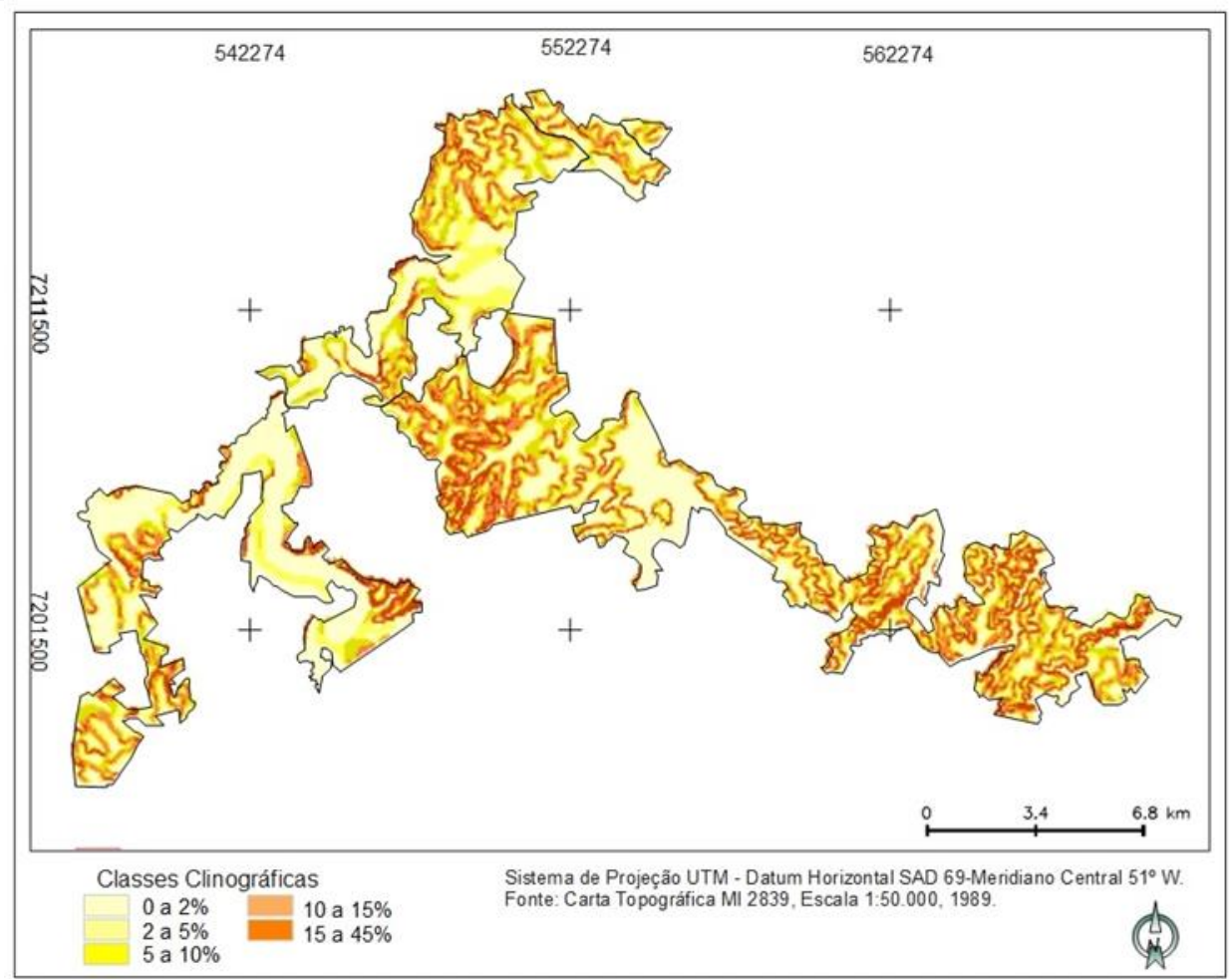

Figura 4: Slope, REBIO

Organizado: Maganhotto, R.F.

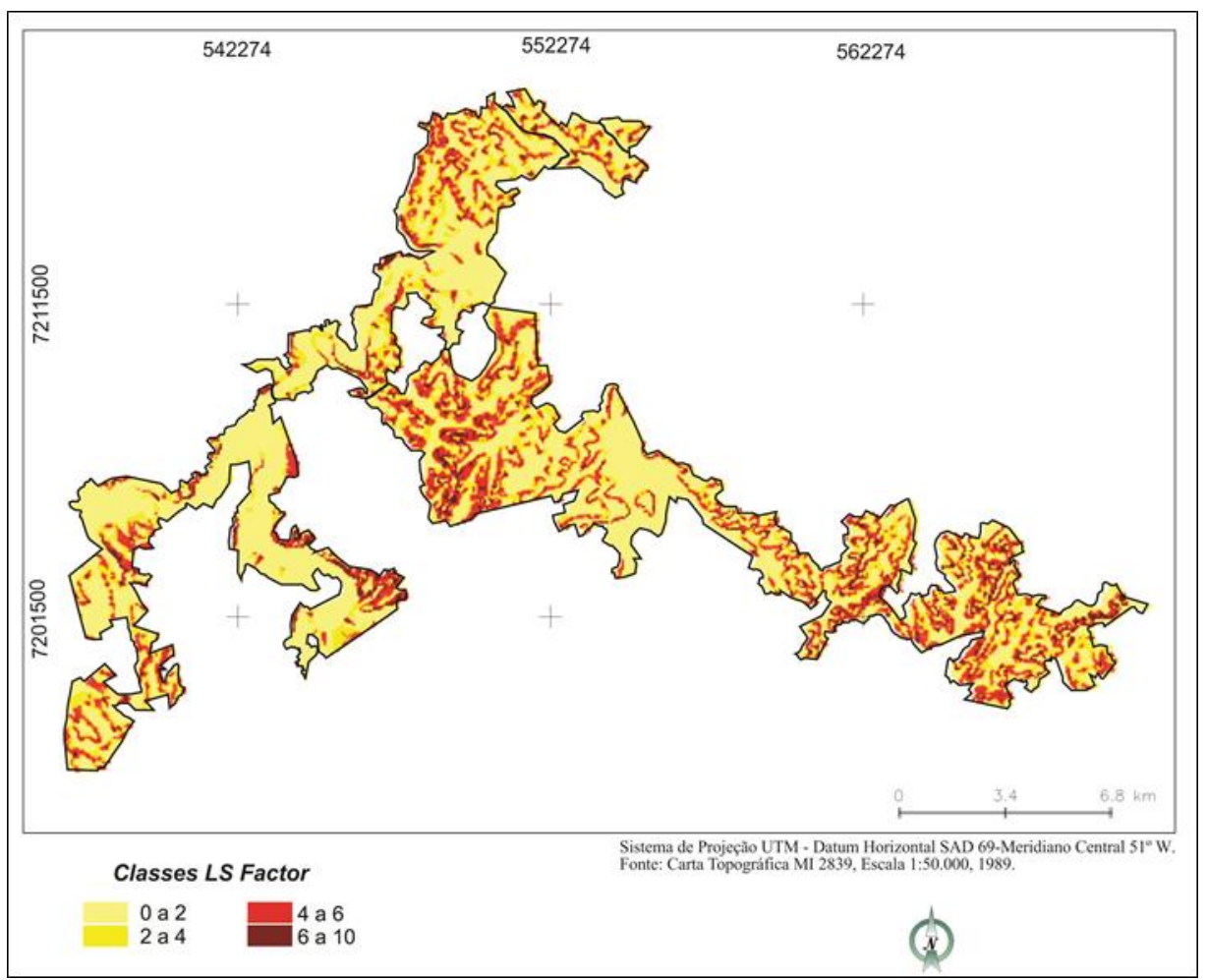

Figura 5: LS REBIO

Organizado: Maganhotto, R.F. 
XVII Simpósio Brasileiro de Geografia Fisica Aplicada

I Congresso Nacional

de Geografia Física
OS DESAFIOS DA GEOGRAFIA FÍSICA NA FRONTEIRA DO CONHECIMENTO

Instituto de Geociências - Unicamp

Campinas - SP

28 de Junho à 02 de Julho de 2017

\section{Aplicação da Lógica Fuzzy e Análise por Multiplos Critérios na REBIO das Aruacárias}

Após análise das variáveis e entendimento de uma representação adequada para cada atributo, foi definido a tipologia de função (F), (linear, j-amoldado, sigmoidal), a curva (C), (crescente ou decrescente) e o ponto de inflexão (PI). Os dados foram reclassificados em uma escala contínua de 255 níveis, onde os valores próximos a 0 representam as condições não desejadas e os valores próximos a 255 assumem condições mais adequadas. A Tabela 1 descreve os critérios adotados à Lógica Fuzzy.

Tabela 1- Parâmetros adotados na Lógica Fuzzy.

\begin{tabular}{|c|c|c|c|c|}
\hline IRR & F/ C /PI & CARACTERIZAÇÃO & $\begin{array}{c}\text { CONDIÇÃO EM } \\
\text { CAMPO }\end{array}$ & REFERÊNCIA \\
\hline AACN & $\begin{array}{l}\text { j-amoldado/ } \\
\text { crescente/ } 2 \text {. }\end{array}$ & $\begin{array}{c}\text { Até } 2 \text { - valor } 0 . \\
\text { Acima de } 2 \text { - crescente até } 255 .\end{array}$ & $\begin{array}{l}\text { Até } 2 \text { - áreas sujeitas a } \\
\text { constantes inundações }\end{array}$ & $\begin{array}{c}\text { Maganhotto } \\
(\mathbf{2 0 1 3})\end{array}$ \\
\hline TWI & $\begin{array}{l}\text { j-moldado/ } \\
\text { decrescente/ } \\
8 .\end{array}$ & $\begin{array}{l}\text { Até } 8 \text { - valor } 255 \text {. Acima de } 8 \text { - } \\
\text { há um decréscimo até } 0 .\end{array}$ & $\begin{array}{l}\text { Acima de } 8-\text { solos } \\
\text { saturados. }\end{array}$ & Prates (2010). \\
\hline SLOPE & $\begin{array}{c}\text { j-moldado/ } \\
\text { decresc./ } \\
15 \% \text {. }\end{array}$ & $\begin{array}{l}\text { Até } 15 \% \text { - valor } 255 \text {. Acima de } \\
15 \% \text { - há um decréscimo até } 0 .\end{array}$ & $\begin{array}{c}\text { Acima de } 15 \%- \\
\text { condições mais } \\
\text { restritivas. }\end{array}$ & Lepch (1991). \\
\hline MIVBF & $\begin{array}{c}\text { j-moldado/ } \\
\text { simétrico/0,5 } \\
\text { e 2,5. }\end{array}$ & $\begin{array}{c}\text { Até } 0,5 \text { - assumem uma } \\
\text { crescente até } 255 \text {. De } 0,5 \text { a } 2,5 \text { - } \\
\text { valor } 255 \text {. } \\
\text { Acima de } 2,5 \text { - decréscimo até } 0 .\end{array}$ & $\begin{array}{l}\text { Até } 0,5 \text { - associados a } \\
\text { erosão. Acima de } 2,5 \text { - a } \\
\text { sedimentação. }\end{array}$ & $\begin{array}{c}\text { Mckenzie e } \\
\text { Gallant (2007). } \\
\text { Maganhotto et al. } \\
(\mathbf{2 0 1 3 )} . \\
\end{array}$ \\
\hline $\mathbf{L S}$ & $\begin{array}{c}\text { j-moldado/ } \\
\text { decrescente/4. }\end{array}$ & $\begin{array}{c}\text { Até } 4 \text { - valor } 255 \text {. Acima de } 4 \text { - } \\
\text { há um decréscimo até } 0 .\end{array}$ & $\begin{array}{c}\text { Acima de } 4 \text { - propensão a } \\
\text { erosão. }\end{array}$ & $\begin{array}{c}\text { Mansor et al. } \\
(\mathbf{2 0 0 2})\end{array}$ \\
\hline
\end{tabular}

Organizador: Maganhotto (2016).

\section{Determinação dos pesos e importância relativa}

Objetivando a identificação dos graus de fragilidade ambiental da REBIO, foi definido como os IRR devem ser combinados entre si. Este processo, além de permitir o estabelecimento do fator de maior significância, demonstra quanto cada um é mais importante que os demais e como os fatores compensam uns aos outros.

O processo resulta em um peso para cada fator e uma avaliação de consistência da comparação pareada. A razão de consistência (ConsistencyRatio - CR) informa ao usuário sobre inconsistências ocorridas durante a atribuição dos pesos. Segundo Saaty e Vargas (1991), a CR não pode ser superior a 0,1 , caso ocorra faz-se necessário reavaliar o processo. Os pesos resultantes da comparação e a razão de consistência encontram-se dispostas na Tabela 2.

O maior peso para o MRVBF se justifica por sua relação com a erosão e sedimentação. A identificação de áreas suscetíveis à erosão e à deposição auxilia na determinação de zonas mais restritivas destinadas ao uso indireto dos recursos naturais. 


\section{OS DESAFIOS DA GEOGRAFIA FÍSICA NA FRONTEIRA DO CONHECIMENTO \\ Instituto de Geociências - Unicamp \\ Campinas - SP \\ 28 de Junho à 02 de Julho de 2017}

O Ls Factor, como produto da relação entre a declividade e comprimento de rampa vem por segundo, configurando-se como um indicativo de áreas com baixo, médio e alto potencial erosivo (MANSOR et al., 2002).

Tabela 2: Valores estabelecidos na comparação pareada

\begin{tabular}{c|c|c|c|c|c|c|}
\hline IRR & MRVBF & TWI & LS & AACN & SLOPE & PESOS \\
\hline MRVBF & 1 & & & & & 0,3228 \\
\hline TWI & $1 / 2$ & 1 & & & & 0,2916 \\
\hline LS & 1 & 2 & 1 & & & 0,1536 \\
\hline AACN & $1 / 2$ & 1 & $1 / 2$ & 1 & & 0,1536 \\
\hline SLOPE & $1 / 5$ & $1 / 2$ & $1 / 3$ & $1 / 2$ & 1 & 0,0784 \\
\hline \multicolumn{7}{c|}{ Razão de Consistência: 0.00 (aceitável) } \\
\hline
\end{tabular}

Fonte: Software Idrisi Andes.

O TWI e o AACN tiveram mesmo peso, por se tratarem de variáveis complementares na indicação de áreas sujeitas ao acúmulo de água e a inundações. Ao associar estes fatores a declividade pode-se identificar áreas com solos mal drenados, além disso, por estarem relacionados à drenagem auxiliam no planejamento de programas de conservação da mata ciliar e também, como os demais atributos citados, na prevenção de assoreamentos dos rios.

Apesar de sua influência direta sobre o processo erosivo, a Declividade teve menor peso para não favorecer áreas planas relacionadas à deposição como as várzeas com alto índice de umidade e acúmulo de água. Entende-se que o relevo plano não garante a inexistência de limitações de uso.

\section{$5 \quad$ Análise por múltiplos critérios}

O último passo no processo de agregação dos fatores foi a aplicação da regra de decisão, utilizando-se o método de agregação por múltiplos critérios (MultiCriteriaEvaluation - MCE) através da Combinação Linear Ponderada (Weight Linear Combination - WCL), onde cada fator é multiplicado por seu peso, resultando em um mapa variando de 0 a 255 , onde os valores próximos a 0 apresentam locais com limitação alta e os valores próximos ou iguais a 255 apresentam áreas de limitação baixa. Com a análise visual do histograma da imagem, verificou-se a distribuição dos valores em quatro grupos, fato que fundamentou a reclassificação da imagem em 4 classes, denominadas de Classes com Limitação Muito Baixa, Baixa, Média e Alta. A Tabela 3 e a Figura 6 apresentam a disposição destas classes na unidade, enquanto a Tabela 4 descreve os valores máximos, mínimos e médios dos IRR nas Classes Limitantes.

A área da REBIO das Araucárias apresenta a maior parte da sua área classificadas como de limitação muito baixa e baixa $(49,00$ e 21,07\%, respectivamente - Tabela 8$)$, principalmente na porção central e leste da área (Figura 6). As áreas classificadas como de limitação muito baixa têm como característica 
XVII Simpósio Brasileiro de Geografia Fisica Aplicada

I Congresso Nacional de Geografia Física

\section{OS DESAFIOS DA GEOGRAFIA FÍSICA NA FRONTEIRA DO CONHECIMENTO \\ Instituto de Geociências - Unicamp \\ Campinas - SP \\ 28 de Junho à 02 de Julho de 2017}

AACN médio de 13,08 m, ou seja, uma distância vertical relativamente grande em relação ao canal de drenagem e consequentemente do lençol freático.

Tabela 3. Classes de limitação ambiental, com a respectiva área e sua proporção a área total da REBIO.

\begin{tabular}{|c|c|c|c|c|c|}
\hline Classes & Área (ha) & $\%$ & Classes & Área (ha) & $\%$ \\
\hline Muito Baixa & 7311 & 49 & Média & 2335 & 15 \\
\hline Baixa & 3142 & 21 & Alta & 2132 & 14 \\
\hline \multicolumn{4}{|c|}{ Total } & 14920 & 100 \\
\hline
\end{tabular}

Fonte: Maganhotto, 2017.

Por conta disso, essas áreas pertencentes a essa classe não sofrem com encharcamentos ou empossamento de água. A declividade média de aproximadamente 4,5\% indicam áreas em que o escoamento lateral de água ocorre de forma suave, sem tendência a processos erosivos. Este fato corrobora os valores de MRVBF médio de 1,31 (Tabela 4) o qual poderia ser interpretado individualmente como área mal drenada, caso a mesma não se situasse tão distante do canal de drenagem (PRATES et al, 2012). O baixo valor médio de LS sugere que as áreas dessa classe não estão sujeitas a processos erosivos intensos, justificando a inclusão da mesma como de muito baixo risco ambiental, ou de degradação (MANSOR et al. 2002).

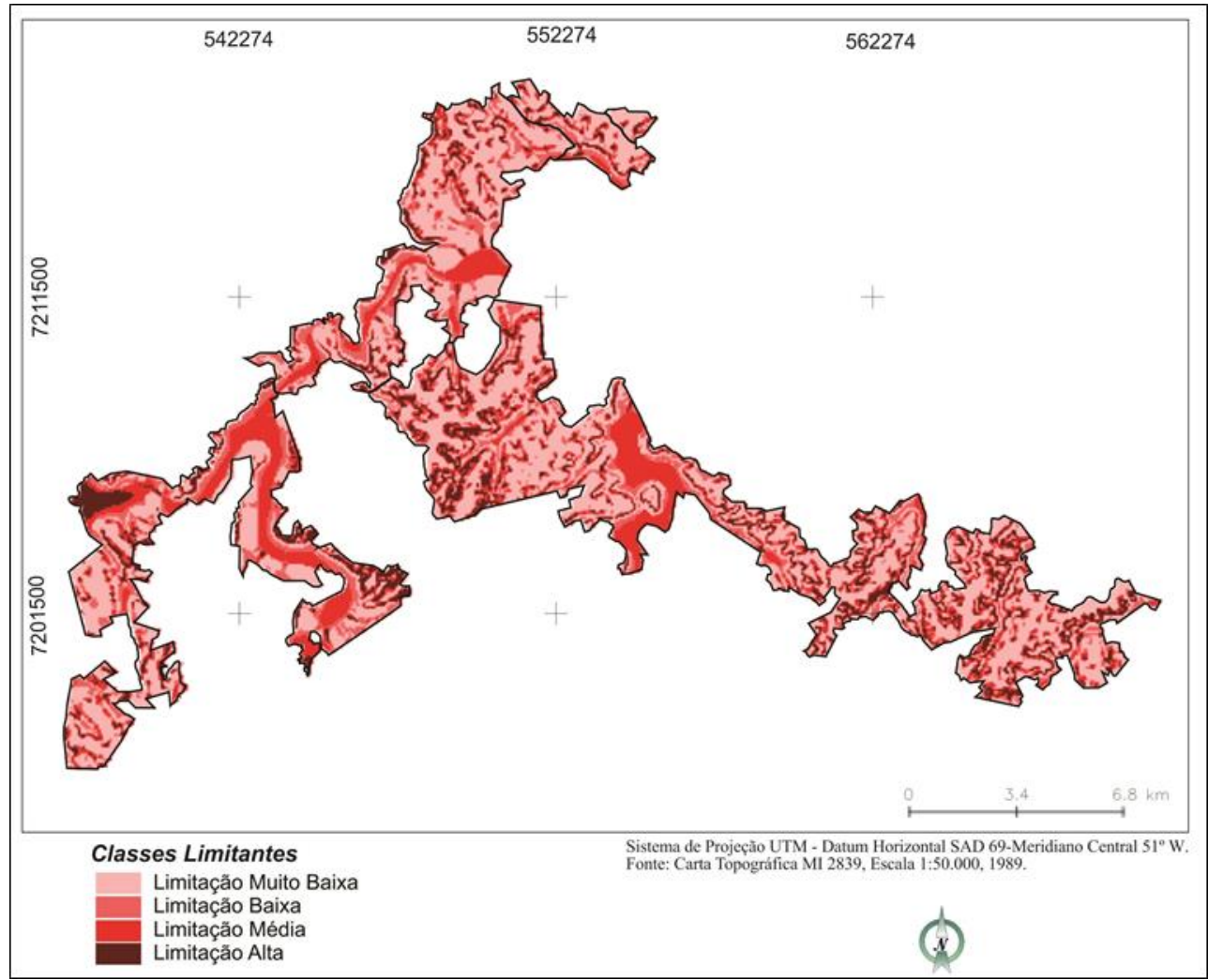

Figura 6: Classes de Limitação

Organizado: Maganhotto, R.F.

Tabela 4:Estatística básica para os valores dos IRR para cada Classe de Limitação Ambiental.

\begin{tabular}{ccccccccc}
\hline & \multicolumn{4}{c}{ Limitação Muito Bixa } & \multicolumn{4}{c}{ Limitação Baixa } \\
\hline IRR & Mín. & Máx. & Média & Desvio & Mín. & Máx. & Média & Desvio \\
\hline AACN & $\mathbf{0 , 0 0}$ & $\mathbf{5 9 , 0}$ & $\mathbf{1 3 , 0 8}$ & $\mathbf{1 2 , 5 7}$ & $\mathbf{0 , 0}$ & $\mathbf{6 4 , 5}$ & $\mathbf{5 , 6}$ & $\mathbf{8 , 8}$ \\
\hline
\end{tabular}




\begin{tabular}{|c|c|c|c|c|c|c|c|c|}
\hline \multirow{2}{*}{\multicolumn{2}{|c|}{$\begin{array}{l}\text { XVII Simpósio Brasileiro } \\
\text { de Geografia Fisica Aplicada } \\
\text { I Congresso Nacional } \\
\text { de Geografia Física }\end{array}$}} & \multirow{2}{*}{\multicolumn{7}{|c|}{$\begin{array}{c}\text { OS DESAFIOS DA GEOGRAFIA FÍSICA NA FRONTEIRA DO CONHECIMENTO } \\
\text { Instituto de Geociências - Unicamp } \\
\text { Campinas - SP } \\
\text { la } \\
\text { a }\end{array}$}} \\
\hline & & & & & & & & \\
\hline $\mathbf{L S}$ & $\mathbf{0 , 0 0}$ & 5,4 & 1,26 & 1,12 & $\mathbf{0 , 0}$ & 6,8 & 2,1 & 2,0 \\
\hline MRVBF & $\mathbf{0 , 0 0}$ & 5,0 & 1,31 & 1,10 & 0,0 & 7,0 & 2,2 & 1,8 \\
\hline Slope & $\mathbf{0 , 0 0}$ & 19,4 & 4,48 & 3,48 & $\mathbf{0 , 0}$ & 27,0 & 5,4 & 5,6 \\
\hline TWI & 4,4 & 24,2 & 8,5 & 2,1 & 4,2 & 24,2 & 10,1 & 3,3 \\
\hline & \multicolumn{4}{|c|}{ Limitação Média } & \multicolumn{4}{|c|}{ Limitação Alta } \\
\hline AACN & $\mathbf{0 , 0}$ & 63,6 & 5,4 & 8,7 & $\mathbf{0 , 0}$ & 55,6 & $\mathbf{8 , 7}$ & 8,4 \\
\hline $\mathbf{L S}$ & $\mathbf{0 , 0}$ & 13,7 & 2,6 & 2,8 & 0,0 & 151,1 & 10,5 & 6,6 \\
\hline MRVBF & $\mathbf{0 , 0}$ & 7,0 & 3,0 & 2,5 & $\mathbf{0 , 0}$ & 8,0 & 0,6 & 1,7 \\
\hline Slope & $\mathbf{0 , 0}$ & 40,5 & 6,3 & 7,3 & $\mathbf{0 , 0}$ & 73,2 & 18,1 & $\mathbf{8 , 7}$ \\
\hline TWI & 4,2 & 24,2 & 10,2 & 3,4 & 4,0 & 23,3 & 7,9 & 2,2 \\
\hline
\end{tabular}

Fonte: Software Idrisi Andes.

As áreas classificadas como de limitação baixa diferenciaram da anterior por estarem situadas mais próximas ao canal de drenagem, distantes verticalmente 5,6 $\mathrm{m}$ (Tabela 4), mas com valores de declividade relativamente baixos, assim como os valores de MRVBF. O principal fator limitante dessa área seria a umidade da encosta, representada pelos valores de TWI maiores em relação a classe apresentada anteriormente.

Em termos de distribuição espacial da área, essa classe está representada por fragmentos dispersos (Figura 6), geralmente na transição entre as áreas de muito baixa fragilidade e de média fragilidade.

A classe de limitação média perfaz 2.335 hectares da REBIO (15,65\% - (Tabela 3). Sua distinção das outras classes se dá principalmente pelo maior valor de MRVBF que, segundo Mckenzie e Gallant (2007), representam processos de sedimentação intensos, uma vez que essas áreas predominam no sopé da encosta, recebendo sedimentos transportados de toda vertente. Os valores de TWI de 10 seriam suficientes para caracterizar esse ambiente como mal drenado (LIN et al., 2006, PRATES et al., 2012). Apesar dos valores médio de AACN e TWI não apresentarem grande diferença para a classe de baixa limitação, é possível observar nas figuras 2 e 3 que as áreas enquadradas nessa classe coincidem com aquelas mais próximas ao canal de drenagem e com maiores valores de TWI. Essa pequena variação dos valores médios se deve, provavelmente, a classe de baixa limitação situar-se na transição entre outras duas classes, como mencionado anteriormente.

As áreas classificadas como de grande fragilidade ambiental somam 2.132 hectares, perfazendo $14,28 \%$ da REBIO. Declividade e LS foram os IRR que apresentaram grande variação em relação as demais classes e consequentemente foram os determinantes para a enquadramento dessas áreas como tal. A média dos valores de declividade para a classe foi de $18 \%$, condição em que predomina o movimento lateral da água, com reduzida infiltração no perfil e potencializando os processos erosivos. Este último corrobora com os elevados valores de LS, o qual também considera comprimento de rampa além da declividade. 
Apesar dos valores médios indicarem a predominância dessa classe em áreas com intenso processo erosivo, é possível que a oeste da REBIO há pequena região classificada como de elevada fragilidade (Figura 6), porém, quando observadas as Figuras 1, 2 e 3, percebe-se que elevados valores de MRVBF e TWI associados e pequenos valores de $\mathrm{AACN}$, os quais sugerem uma área mal drenada, provavelmente com sérios problemas de saturação hídrica. Esta área pode influenciar as médias discutidas anteriormente, em que os valores de LS e Declividade podem estar sendo subestimados, visto que ambas áreas associadas a elevada fragilidade ambiental, são geomorfologicamente distintas.

Pode-se afirmar que o processamento dos IRR e a aplicação da Lógica Fuzzy, possibilita a avaliação conjunta destes atributos, facilitando o entendimento da dinâmica natural da unidade, fundamentando o planejamento ambiental e a gestão da referida unidade de conservação.

\section{Considerações Finais}

A aprovação de um decreto não garante a conservação da natureza, as unidades de conservação (UC's) apresentam dificuldades relacionadas a recursos humanos e financeiros; a existência de atividades conflitantes em seu perímetro e entorno; a regularização fundiária; e a inexistência do Plano de Manejo.

Neste sentido, as metodologias de planejamento subsidiando a gestão racional das UC's, devem caracterizar os elementos naturais, contemplando critérios e procedimentos metodológicos relacionados aos aspectos geomorfológicos, clinográficos e pedológicos, contribuindo para que a intervenção humana não venha a desencadear e ou acelerar problemas de ordem ambiental..

Com base nos dados e informações levantadas, pode-se afirmar que, o processamento e aplicação dos IRR mostraram-se adequados ao planejamento da unidade tanto como instrumento auxiliar na delimitação e estabelecimento das zonas, quanto no planejamento e manejo das atividades previstas para a REBIO.

\section{Agradecimentos}

Agradecemos a Fundação Araucária e a Fundação O Boticário, instituições estas, financiadoras da presente pesquisa.

\section{Bibliografia}

BERTONI, J; LOMBARDI NETO, F. Conservação do solo. São Paulo: Ícone, 1990. 355p.

BÖHNER, J.; KÖTHE, R.; CONRAD, O.; GROSS, J.; RINGELER, A.; SELIGE, T. Soil Regionalization by Means of Terrain Analysis and Process Parameterisation. In: MICHELI, E.; NACHTERGALE, F.; 
MONTANARELLA. L. (Ed.). Soil Classification 2002. European Soil Bureau - Research Report, n. 7, EUR 20398 EN, Luxembourg, p. 213-222, 2002.

BURROUGH, P. A. Principles of geographical information systems of land resources assessment. Francis e Taylor, 1986. 185 p.

CARVALHO-FILHO, L.M., XAVIER-DA-SILVA, J. \& Almeida, L.F.B. Methodology for Data Processing Aiming the GIS Input. Joint European Conference and Exhibition on Geographical Information, The Hague, Proceedings, Volume 1, p. 30-35, 1995.

GALlANT, J. C.; WILSON, J. P. "Primary topographic attributes". In: WILSON, J. P.; GALLANT, J. C. (Eds.). Terrain Analysis: Principles and applications. New York: John Wiley, 2000.

GRUBER, S.; PECKHAM, S. Land-surface parameters and objects in hydrology. In: HENGL, T.; REUTER, H.I. (Eds.). Geomorphometry - Concepts, Software, Applications. Amsterdam: Elsevier, 2009.

IPPOLITI, R. G. A.; COSTA, L. M.; SCHAEFER, C. E. G. R.; FERNANDES FILHO,E. I.; GAGGERO, M. R.; SOUZA, E. Análise digital de terreno: Ferramenta na identificação de pedoformas em microbacia na região de "mar de morros" (MG). Revista Brasileira de Ciência do Solo, Viçosa, v. 29, n. 2, p. 269-276, 2005.

LEPCH, I. F. (Coord.). Manual para levantamento utilitário do meio físico e classificação de terras no sistema de capacidade de uso. $4^{\mathrm{a}}$ aproximação, $2^{\mathrm{a}}$. Imp. rev. Campinas: Sociedade Brasileira de Ciências do Solo, 1991.

LIN, H.S.; KOGELMANN, W.; WALKER, C.; BRUNS, M.A. Soilmoisturepatterns in a forestedcatchment: A hydropedological perspective. Geoderma, v. 131, p. 345-368, 2006.

MAGANHOTTO, R. F. et al. Os Índices de Representação do Relevo como Suporte para o Zoneamento Ambiental de Unidades de Conservação - Estudo de Caso da Floresta Nacional de Irati.Geografia, Ensino \& Pesquisa, Vol. 20 (2016), n.3, p. 168-181

MAGANHOTTO, R. F.; SANTOS, L. J. C. dos; SOUZA, L. C. de P. Miara. M. A.; LEMES, P. H. S. “A aplicação dos Índices de Representação do Relevo como ferramenta de suporte no planejamento ambiental de unidades de conservação". Revista Geografar, v. 8, n. 2, p. 205 - 236, Curitiba, 2013.

MANSOR, M.T.C.; FERREIRA; L.; ROSTON, D.M.; TEIXEIRA FILHO J. Parâmetro para avaliação do potencial de risco de erosão. In: Simpósio Regional De Geoprocessamento E Sensoriamento Remoto, I., 2002. Anais... Aracaju/SE, 17 e 18 de outubro de 2002.

MOORE, I. D.; GESSLER, P. E.; NIELSEN, G. A.; PETERSON, G. A. Soil attribute prediction using terrain analysis.Soil Science Society of America Journal, Madison, v. 57, n. 2, p. 443-452, 1993.

MCKENZIE, N. J.; GALLANT, J. C. "Digital soil mapping with improved environmental predictors and models of pedogenesis". Developments in Soil Science, v. 31, 2007. 
MCKERGOW, L.A.; GALLANT, J.C.; DOWLING, T.I. "Modelling wetland extent using terrain indices, Lake Taupo", NZ. In: InternationalCongressonModellingandSimulation, Christchurch, 10-14 December 2007.

PRATES, V. Utilização de índices para representação da paisagem como apoio para levantamento pedológicos em ambiente de geoprocessamento. Dissertação (Mestrado) - Programa de Pós Graduação em Ciências do Solo, Universidade Federal do Paraná,UFPR, Curitiba, 2010.

ROSS, Jurandyr L. Sanches. Ecogeografia do Brasil: subsídios para o planejamento ambiental. São Paulo: Oficina de Textos, 2006. 